MATEC Web of Conferences 44, 01065 (2016)

DOI: $10.1051 /$ matecconf/20164401065

(C) Owned by the authors, published by EDP Sciences, 2016

\title{
Design and Experiment of Flight Path Control System of Unmanned Autogyro
}

\author{
Song Wang ${ }^{1, a}$, Wan Li Chen², Wen Qiang Dun², Liang Liang Bu² and Fang Chen Dong ${ }^{2}$ \\ ${ }^{1}$ UAV Research Institute, Beihang University, Beijing 100191, China \\ ${ }^{2}$ Robotics Institute, School of Mechanical Engineering \& Automation, Beihang University, Beijing, 100191, China
}

\begin{abstract}
This paper presents the architecture of flight tracking control system for unmanned autogyro, and designs the control law based on the control characteristics of unmanned autogy-ro. To reduce the lift force loss during turning and maintain the altitude, the feedforward control method of pitching rudder for propeller is adopted (dur-ing rolling); To reduce the influence of propeller anti-twisting effect on the roll at-titude and course, feedforward control method of the propeller rolling rudder (during throttle changing) is adopted; to reduce the slide slipping and achieve coordinated turning, a hybrid control strategy of the yaw rudder and rolling rud-der of propeller is developed. The flying platform is built to verify the flying. It is proved that this control system can effectively control the altitude and horizontal path of the unmanned autogyro, the control accuracy is better than $\pm 5 \mathrm{~m}$.

Keywords. Autogyro UAV; flight path control; control law; feedforward control; flight verification
\end{abstract}

\section{Foreword}

The autogyro is an aircraft which gains the lift force via the main rotor of the free rotation, and gains the thrust from the propeller; it features short take-off and landing distance, simple structure, low cost, high safety and other advantages. In recent years, as the UAV (Unmanned Aerial Vehicle) industry booms, the unmanned autogyro becomes a new research focus.

United States Navy once conducted the research on unmanned autogyro and the wind tunnel test; scholars of University of Maryland conducted the relevant test and study on the run-up mechanism and characteristics of nonmotile rotor of the autogyro, and achieved certain results [1]; Sweden also developed a midget unmanned autogyro to equip the Sweden Armed Forces [2]. Comparatively, domestic study on autogyro UAV is rare. The Aviation Simulation and Control Laboratory of Nanjing University of Aeronautics and Astronautics once used a prototype as the platform to conduct the research regarding flight control [3]; Wang Deshuang and other experts of AVIC Aerodynamics Institute have designed a semi-physical simulation system for autogyro flight control [4].

The development of flight control technologies of unmanned autogyro in China is basically at the phase of theoretical and semi-physical simulation. The actual flight has not been reported yet, which poses an opportunity and challenge for the work in this paper. This paper presents a design for flight path control system that suits autogyro characteristics, and conducts flight verification on the small-scale test prototype, which achieves the altitude control and flight path tracking and can provide reference for the flight control system design of medium and large unmanned autogyro in the next stage.

\section{Control system architecture}

The unmanned autogyro control system achieves the attitude stabilization, position tracking and velocity tracking of the gyroplane via the control of the propeller horizontal rolling rudder, pitching rudder [9], yaw rudder and throttle. The horizontal rolling and pitching are achieved via the linkage of the two steering engines.

When the two controlling steering engines work with counter actions, they complete the horizontal rolling function, which is similar to the aileron of the fixed wing UAVs; when the two control steering engines work with equidirectional actions, they complete the pitching function, which is similar to the elevator of the fixed wing UAVs.

The flight control requirement of gyroplane is similar to the fixed wing UAVs, but the customized controller structure and control laws should be specially designed according to the characteristics of gyroplane.

The flight path control system for autogyro includes the sensor system, master control system and servo actuation system etc. Its system architecture is shown in Figure 1.

\footnotetext{
${ }^{a}$ Song Wang: wsongking@126.com
} 


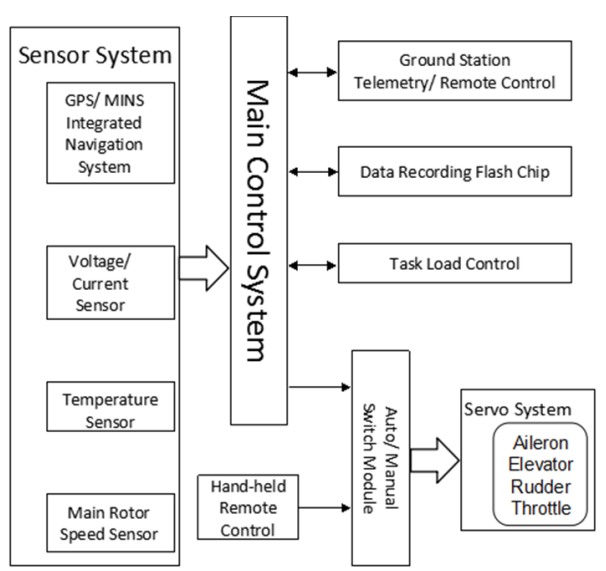

Figure 1. Autogyro flight path control system architecture

In this architecture, the master control system is at the core position, which links the other parts closely to jointly achieve the following functions [5]:

- Real-time measurement of the posture, position, altitude, velocity, angular rate and acceleration etc. of the gyroplane and the navigation computing;

- Real-time monitoring of the voltage of the main power supply, steering engine voltage, main rotor rotation speed and machine room temperature and other signal information related to the flight safety as well as complete malfunction alarm and emergency protection mechanism;

- Online adjusting the paraments of control law via the ground station;

- Synchro recording the key state variables and the operating instruction during the flight;

- Switches between the manual and auto mode via the remote controller;

- Controlling of the output of horizontal rolling rudder and pitching rudder of the propeller, the yaw rudder, throttle, etc.

The physical figure and the technical parameters of the flight path control system is shown in Figure 2 and Table 1 .

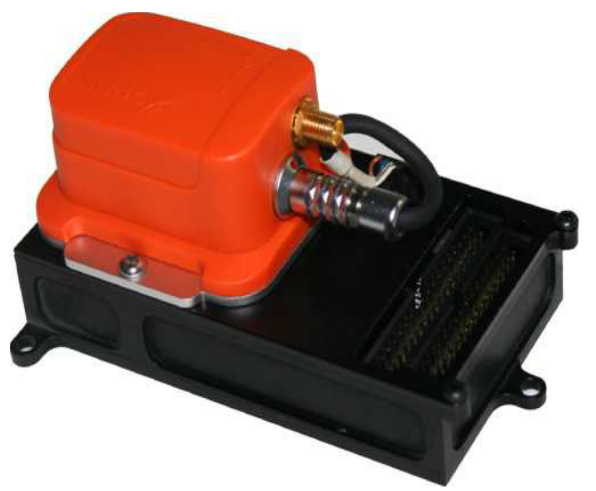

Figure 2. Physical map of the gyroplane flight tracking control

Table 1. Specifications of the autogyro flight tracking

\begin{tabular}{|c|c|}
\hline Size & $110 \mathrm{~mm} \times 58 \mathrm{~mm} \times 53 \mathrm{~mm}$ \\
\hline Weight & $200 \mathrm{~g}$ \\
\hline Power consumption & $700-1350 \mathrm{~mW}$ \\
\hline
\end{tabular}

\section{The design of control law}

The control channels of autogyro include the longitudinal channel, lateral passage, speed channel, etc. The longitudinal channel controls the angle of pitch and altitude via the propeller pitching rudder; the lateral passage controls the roll position, course and horizontal position via the horizontal rolling rudder and the yaw rudder; the speed channel controls the speed by adjusting the propeller rotation speed [6].

Control laws for each channel are designed in this paper based on the flight control characteristics of the autogyro, with details expounded as follows:

\subsection{Longitudinal channel}

The longitudinal channel adopts the cascade PID controller, with which outer loop controls altitude, and inner loop controls pitch attitude. The control method is based on the altitude difference, the altitude controller calculates the target pitch angle, and inputs it to the pitch stabilization and control circuit, and calculates and outputs the pitching rudder factor to control the gyroplane pitch attitude and correct the altitude.

The characteristic of longitudinal channel control is that the gyroplane altitude falls due to the fact that the propeller vertical pulling force cannot offset the counterweight force, and the propeller pitches laterally during the bank-and-turn in the forward flight process of the gyroplane [7].

To reduce the lifting force loss during the turning and maintain the altitude, this paper adopts the pitching rudder feedforward control method of the propeller (during rolling), the controller structure is shown in Figure 3:

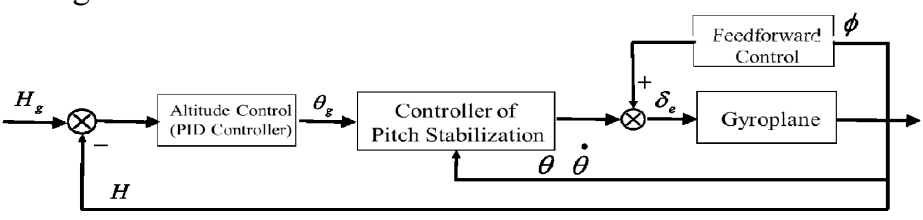

Figure 3. Structure of longitudinal channel controller

In Figure $3, H_{g}$ is the given target altitude; $H$ is the actual altitude; $\theta_{g}$ is the calculated target pitch angle; $\theta$ and $\dot{\theta}$ are the true angle of pitch and true angle of pitch rate respectively; $\phi$ is the actual roll angle; $\delta_{e}$ is the pitching rudder output factor of the propeller.

\subsection{Lateral channel}

The lateral channel also adopts cascade PID controller, with which the external loop controls the level position, and the inner loop controls the roll position and course. The external loop control adopts the lateral deviation guidance method..

The control characteristics of the lateral channel of gyroplane are as follows:

- When controlling the gyroplane to turn, if the yaw rudder only is operated, the gyroplane will have outward slipping; if the rolling rudder of the propeller only is operated, the gyroplane will have inward slipping. To 
reduce the lateral slipping and achieve the coordinated turning, a hybrid control strategy for the rolling rudder is developed by the authors: based on the lateral deviation and the lateral deviation speed, the lateral deviation controller calculates the target course angle and the target rolling angle. The target course angle plus the forward course angle is input to the course stabilization and control circuit, which will calculate and output the yaw rudder value; the target rolling angle are input to the roll stabilization and control circuit, which will calculate and output the rolling rudder value [8]. The yaw rudder and roll rudder of the propeller jointly complete the rolling posture and course control of the gyroplane and correct the lateral deviation.

- The gyroplane generally adopts the back-push propeller, with untwisting effect greater than the front-pull type. In addition, the throttle change may result in the untwisting force change on the gyroplane, which further causes the rolling posture and course fluctuation. Therefore, this paper adopts the rolling rudder feedforward control method (during throttle changing) to offset the untwisting torque.

Based on the above study, the controller structure of the lateral channel of autogyro is shown in Figure 4.

$$
D \dot{D}
$$

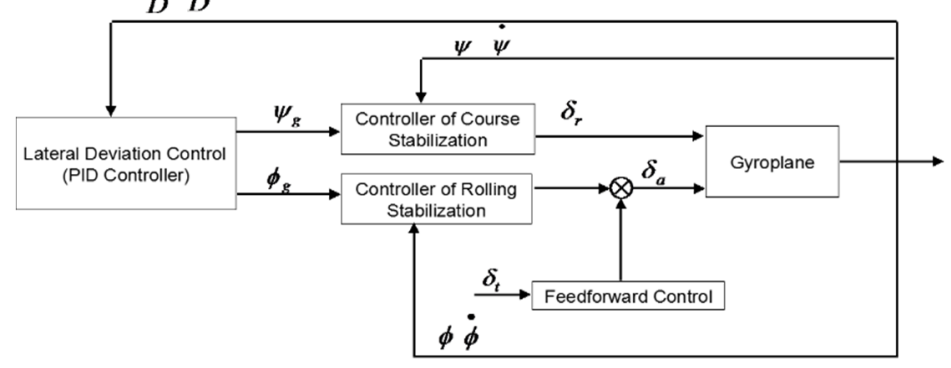

Figure 4. Structure of lateral channel controller

In Figure $4, D$ and $\dot{D}$ are the lateral deviation and lateral deviation speed respectively; $\psi_{g}$ and $\phi_{g}$ calculate the target course angle and target rolling angle separately; $\psi, \dot{\psi}, \phi$ and $\dot{\phi}$ are the true course angle, the actual course angular rate, actual rolling angle and the actual rolling rate respectively; $\delta_{t}$ is the throttle rudder value; $\delta_{r}$ and $\delta_{a}$ are output yaw rudder value and the rolling rudder value respectively.

\subsection{Speed channel}

The structure of the speed channel controller is shown in Figure 5:

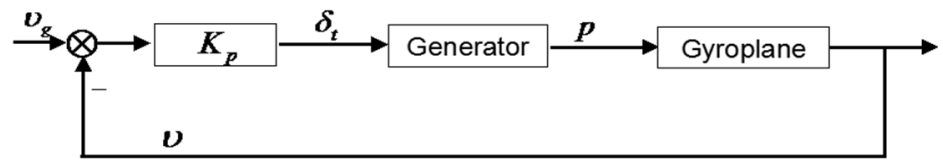

Figure 5. Structure of speed channel controller

In Figure 5, $v_{g}$ is the target airspeed; $K_{g}$ is the proportional component gain; $\delta_{t}$ is the throttle rudder value; $p$ is the propeller thrust; $v$ is the true air speed.

The small-scale test prototype studied in this paper is not installed with the airspeed sensor, so a simplified speed controller is applied during the flight test.
As the main rotor of the autogyro is nonmotile wind power rotor, it has such disadvantages as obvious lagging and non-linearity in driving the rotor [9]. The control strategies for speed channel accordingly developed in this paper are as follows:

$$
\left\{\begin{array}{c}
\delta_{t}=\delta_{t 0}, \text { when } \theta_{g}<0 \text { and } \theta<0 \\
\delta_{t}<\delta_{t 2}, \text { when } \theta_{g}>0 \text { and } \theta>0 \\
\delta=\delta_{t 1}, \text { other }
\end{array}\right.
$$

In the above formula, $\delta_{t 0}, \delta_{t 1}$ and $\delta_{t 2}$ are the diving throttle, cruise throttle and climbing throttle respectively, which are given during calibration; $\theta_{g}$ and $\theta$ are target pitch angle and true angle of pitch respectively.

\section{Experiment verification}

During the experiment of this paper, a minitype electric drive gyroplane is installed with the flight path control system and data transmission module. The physical figure and the technical parameters of this prototype is shown in Figure 6 and Table 2:

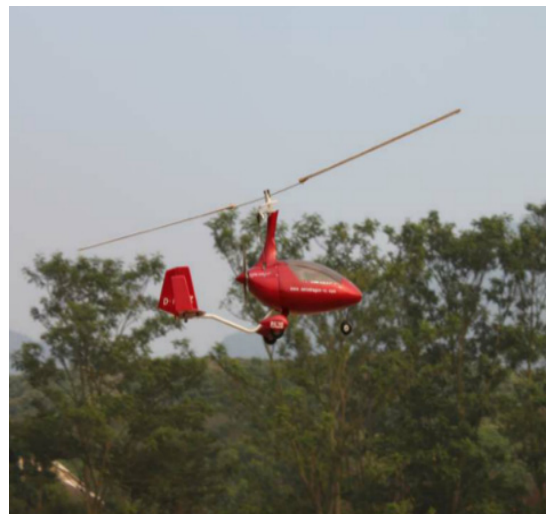

Figure 6. Physical map of the prototype

\begin{tabular}{|c|c|}
\hline Rotor DIA. & $1840 \mathrm{~mm}$ \\
\hline Body length & $1048 \mathrm{~mm}$ \\
\hline Body height & $600 \mathrm{~mm}$ \\
\hline Propeller & $13 \mathrm{inch} \times 6.5 \mathrm{inch}$ \\
\hline TOW. & $3500 \mathrm{~g}$ \\
\hline Flying speed & $10-100 \mathrm{~km} / \mathrm{h}$ \\
\hline Take-off mode & $\begin{array}{c}\text { Taxi take off } \\
\text { (Distance } \leq 10 \mathrm{~m})\end{array}$ \\
\hline Landing mode & $\begin{array}{c}\text { Taxi landing } \\
\text { (Distance } \leq 10 \mathrm{~m})\end{array}$ \\
\hline
\end{tabular}

Table 2. Specifications of the prototype

The experimental process followed in this paper is: first, the ground station uploads the flight course via the wireless link circuit; then manually controls the gyroplane to take off; after reaching certain altitude, it switches to auto flight mode, and the gyroplane completes the auto flight as per the scheduled course via the flight path control system; during the flight, the ground station sends the flight course change instruction to test the various performance factors. 
This experiment includes altitude control performance test, horizontal path control performance test and quadrilateral flight course test.

\subsection{Performance test of altitude control}

The performance test of altitude control is conducted by switching between two flight courses. One is $80 \mathrm{~m}$-high quadrilateral flight course, and the other is $105 \mathrm{~m}$-high quadrilateral flight course. This link consists of altitude tracking of climbing and descending branch stage, with the test results shown in Figure 7 and 8:

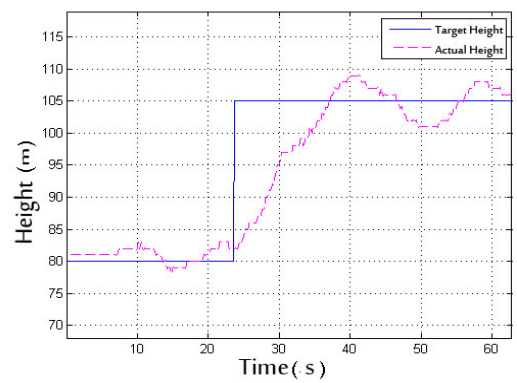

Figure 7. Height tracking curve in climbing test

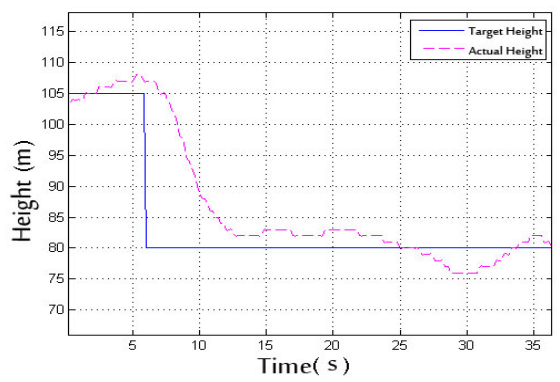

Figure 8. Height tracking curve in diving test

\subsection{Performance test for horizontal path control}

In this test, the gyroplane starts to converge to the planned flight course at the $45 \mathrm{~m}$ lateral deviation point; the lateral deviation convergence curve is shown in Figure 9:

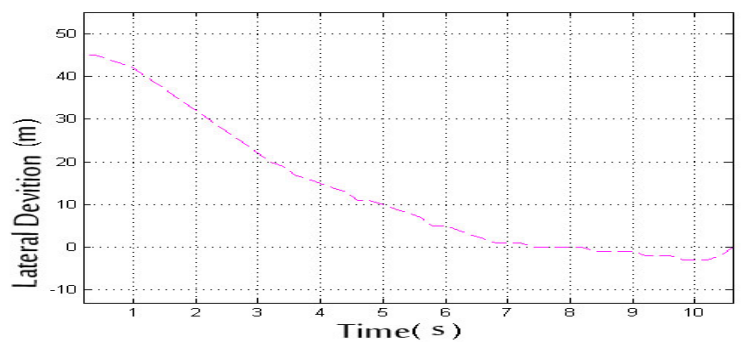

Figure 9. Lateral offset convergence curve

It can be seen that, the convergence procedure is smooth, from $45 \mathrm{~m}$ to 0 , it only takes about $7 \mathrm{~s}$, and the speed of convergence is about $6.5 \mathrm{~m} / \mathrm{s}$. After it is on the planned flight course, the lateral deviation curve is smooth and the control accuracy is better than $\pm 5 \mathrm{~m}$, but the lateral deviation is more of the flight course, which is due to the small scope of the flight course, and the lateral deviation has not reached the steady state during the gyroplane turning.

\subsection{Quadrilateral flight course test}

Based on the above control performance tests, a quadrilateral flight course is proposed by the authors, which is $80 \mathrm{~m}$ high, $200 \mathrm{~m}$ long and $80 \mathrm{~m}$ wide. The gyroplane will fly 12 circles under the auto mode, with the total journey of $7 \mathrm{~km}$, and the flight time about 6 minutes. Taking the small battery capacity into consideration, this test is conducted in 3 missions each with 4 flights.

Certain session of test data is selected for analysis, and the results are shown in Figure 10 and 11:

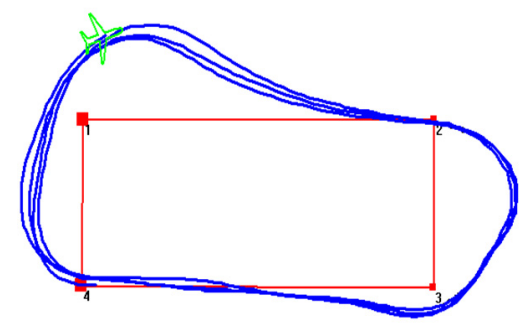

Figure 10. Track map in circular route test

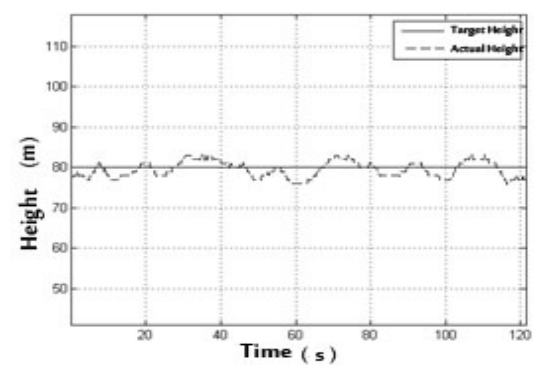

Figure 11. Height tracking curve in circular route test

In it, the rectangle is the planned flight course and the curve is the flight path.

From Figure 10 and 11, it can be seen that the unmanned autogyro can achieve the auto flight as per the planned flight course, with average speed about $20 \mathrm{~m} / \mathrm{s}$; the horizontal path fluctuation is less than $10 \mathrm{~m}$, with high overlap ratio; the altitude control accuracy is better than $\pm 5 \mathrm{~m}$, which shows cyclical changes, due to the fact that the gyroplane turns frequently, and the turning altitude is not stable yet.

There is a big lateral deviation during the switch between navigation point 2 and 4, which is due to the following reasons:

- The gyroplane is incapable of steep turn during the flight;

- The flight course is small; the lateral deviation does not converge to 0 when the gyroplane switches between navigation points.

\section{Conclusion and prospect}

This paper mainly presents the design of flight path control system of unmanned autogyro, and conducts the auto flight of the experimental prototype. It is concluded that this system can effectively control the altitude and 
horizontal path of unmanned autogyro, which has a high degree of precision and reliability.

The key study in the next step is to enhance the sensor precision, further adjust the control laws and other work with a view to achieve the flight control of large and medium unmanned autogyro.

\section{References}

1. Chen M. Research on flight control technologies for unmanned gyroplane [D] Nanjing: Nanjing University of Aeronautics and Astronautics, 2011(in Chinese).

2. Wei K, Li Z G, Ma C W. Technology and development prospects of unmanned gyroplane [J]. Aerodynamic Missile Journal, 2012, (12):37-40 (in Chinese).

3. Chen J. Research on flight control and simulation technology of unmanned gyroplane [D]. Nanjing: Nanjing University of Aeronautics and Astronautics, 2013 (in Chinese).

4. Wang D S, Li G, Yang N. Semi-physical simulation system of a certain type of unmanned gyroplane [J]. Ordnance Industry Automation, 2014, 33 (4):22-25 (in Chinese).

5. Wang S. Study of the integrated flight control system for miniature fixed-wing unmanned aerial vehicles [D]. Beijing: Beihang University, 2010 (in Chinese).
6. Xu H, Zhang B H, et al. Research on control system of small unmanned gyroplane [J]. Science, 2011, (12):145-147 (in Chinese).

7. Hao C J. Research on control technology of unmanned gyroplane [D]. Nanjing: Nanjing University of Aeronautics and Astronautics, 2011 (in Chinese).

8. Chen J, Wang D B, et al. Turning flight control of unmanned gyroplanes based on deviation correction [J]. Ordnance Industry Automation, 2012, 31 (1):5760 (in Chinese).

9. Chen M, Wang D B, et al. Study on navigation control of turning flight for unmanned rotorcraft $[\mathrm{J}]$. Electronics Optics \& Control, 2012, 19 (3):6-11 (in Chinese).

10. Gilbert L. Crouse. Conceptual design of an unmanned gyroplane for an endurance mission[C]//45th AIAA Aerospace Sciences Meeting and Exhibit. Reno, Nevada: AIAA, 2007.

11. Liu W D, Wang D L, et al. MATLAB/Simulink and process control system [M]. Beijing: China Machine Press, 2012:127-143 (in Chinese). 